\title{
Breve análisis de algunos aspectos normativos y técnicos de la evaluación del desem- peño docente para la permanencia del Servicio Profesional Docente
}

A brief analysis of some normative and technical aspects of the appraisal of the teaching performance to keep occupational status into Professional Teaching Service

\author{
David Castro Porcayo (D) \\ e-mail: 1lanero68@live.com.mx \\ Universidad Nacional Autónoma de México. México \\ Roberto Solis González \\ e-mail: rsolis_glz@yahoo.com \\ Universidad Nacional Autónoma de México. México
}

\begin{abstract}
Resumen
En México, la aplicación de la reforma educativa promovida por el gobierno federal en el año 2013 tuvo el propósito de garantizar la profesionalización de la práctica docente considerando el ingreso, desempeño, reconocimiento y promoción docente. Entre las diferentes evaluaciones que se aplican, la evaluación de desempeño para la permanencia se considera como de alto impacto debido a sus implicaciones laborales y políticas. El instrumento ha sido puesto en tela de juicio, en cuanto a su validez, debido a que, por sus características, se asume que la medición resulta limitada para la toma de decisiones laborales de alto impacto sobre el docente. Por lo que, el presente artículo tuvo el propósito de describir sus características y realizar un análisis de la consistencia que existe entre documentos normativos y técnicos. Se observó que existe concordancia entre leyes generales, entre las instituciones que tienen la función de establecer los aspectos normativos y técnicos (Instituto Nacional para la Evaluación Educativa), como la que se encarga de la elaboración de instrumentos, métodos, proceso y aplicación, que es la Secretaría de Educación Pública (SEP). Sin embargo, al comparar los documentos técnicos y los procesos, se encontró que existe una falta de consistencia, en cuanto a las características y propósitos de la evaluación de desempeño, ya que existen diferentes usos y los instrumentos no están del todo adecuados a los tipos de población y condiciones laborales.
\end{abstract}

Palabras clave: evaluación docente; desempeño profesional; condición laboral

\section{Abstract}

In Mexico, the application of the educational reform promoted by the federal government in 2013 was intended to ensure the professionalization of the teaching practice in terms of labour income, performance, recognition and teacher promotion. Among the different Appraisals that are applied, the appraisal for an extended stay into Professional Teaching Service is considered as having a high impact due to its labor and political implications. The instrument has been called into question, in terms of its validity, because, due to its characteristics, it is assumed that measurement is limited for making high-impact labor decisions about the teacher. Therefore, the present article had the purpose of describing its characteristics and performing an analysis of the consistency that exists between normative and technical documents. It was observed that there is agreement between general laws, between the institutions that have the function of establishing the normative and technical aspects (National Institute for Educational Evaluation), such as the one in charge of the elaboration of instruments, methods, process and application, which it is the Secretariat of Public Education (SEP). However, when comparing the technical documents and regulatory processes, it was found that there is a lack of consistency, in terms of the characteristics and purposes of the performance appraisal, since there are different uses and the instruments are not fully adequate for the types of population and working conditions.

Keywords: teacher evaluation; professional performance; employment status

Recibido / Received: 05-09-2018

Aceptado / Accepted: 19-12-2018

Publicación en línea / Published online: 15-05-2019

Cómo referenciar este artículo / How to reference this article:

Castro, D., \& Solís, R. (2019). (En prensa) Breve análisis de algunos aspectos normativos y técnicos de la evaluación del desempeño docente para la permanencia del Servicio Profesional Docente. Tendencias Pedagógicas, 34, pp. 153-168. doi: 10.15366/tp2019.34.012 


\section{Introducción}

En México, con la aplicación de la reforma educativa promovida por el gobierno federal, en el año 2013, se buscó disminuir el control discrecional que ejercían gobiernos y sindicatos sobre las plazas docentes. Específicamente, en el proceso de reclutamiento, selección y contratación de docentes. De los diferentes tipos de instrumentos, que se aplican en cada una de estas etapas, los que miden el Desempeño para la permanencia, son los que más interés y preocupación han suscitado, debido a su alto impacto, ya que están vinculados a la continuidad en el puesto. Si el docente no acredita el examen, puede ser separado de las clases frente a grupo (si ya se encontraba laborando antes de la aplicación de la reforma) o puede ser despedido (si fue contratado después de la reforma).

Con base en lo anterior, el presente estudio tuvo como propósito analizar algunos aspectos normativos y técnicos. Se debe precisar que este documento presenta las limitaciones que resultan de no tener acceso directo a los instrumentos y rúbricas, por lo que, se realizó grosso modo un análisis de la consistencia entre la normativa y algunos aspectos técnicos. A partir de esto, se ofrecen algunas propuestas, que por supuesto, solo pretenden participar de la discusión sobre el tema.

Así, en primer lugar, se establecen algunos referentes teóricos y aspectos laborales sobre los docentes que deben presentar la evaluación de desempeño para la permanencia. En segundo lugar, se describen los instrumentos que compone dicha evaluación. En tercer lugar, se realiza un análisis de la consistencia de algunos aspectos, entre la normatividad y aspectos técnicos. Finalmente, se presentan una serie observaciones que no pretenden ser exhaustivas argumentaciones, si no se procura poner en la discusión aspectos que deben considerarse sobre la evaluación de desempeño docente para la permanencia en el puesto.

\section{Algunos referentes teóricos}

La evaluación de desempeño ayuda a identificar las características del trabajo y el nivel de alcance de la práctica de un docente en el aula. La práctica docente es el proceso por medio del cual, el docente aplica sus habilidades, actitudes o conocimientos que pueden ser de tipo teórico, metodológico y técnico en el ejercicio de su profesión para alcanzar algún propósito. La forma en que se lleva a cabo el desempeño laboral, grosso modo, puede determinarse, por un lado, con base en las características que tienen los procesos implementados para dar cumplimiento a las tareas y funciones propias del puesto y por otro, con el producto alcanzado y su relación con los objetivos.

Estos aspectos han sido abordados por investigadores como Matthews (2013), quien consideró que para evaluar al docente se requiere diferenciar los siguientes aspectos: el nivel que ha alcanzado el docente en la apropiación de saberes sobre la práctica docente; la capacidad que demuestra para usar esos saberes en la resolución de problemas didácticos en el aula y el resultado de su acción sobre el alumno, es decir, el nivel de aprendizaje que logra como producto de la enseñanza. Pero, va más allá y asume que la práctica docente está influenciada por diversos factores y variables (contexto). Para tratar de comprender el fenómeno, seleccionó, a su juicio, las variables más relevantes, dentro del sistema educativo inglés, entre las que se encuentran: la motivación y compromiso del alumno para aprender, el ambiente familiar, rendimiento previo del estudiante; estatus socioeconómico; cohesión en el aula y la gestión escolar.

En cuanto a la interacción en el aula, Coll y Solé (2002) ahondan y consideran que la evaluación enfocada en la práctica docente debe comprender un «triángulo interactivo» que comprende la interacción que existe entre el profesor, los alumnos y el contenido. Implica el análisis de la práctica del profesor que incluye, por un lado, la interactividad que alude al despliegue de acciones que el profesor y los alumnos realizan antes, durante y después de la situación didáctica. Además, añaden que se deben considerar los mecanismos de influencia educativa que se refiere a cómo aprenden los alumnos gracias a la ayuda del profesor. Allal (1980) consideró que la evaluación de la prác tica se debe enfocar en el tipo de acción empleada para realizar adaptaciones al proceso de intervención pedagógica de acuerdo con los problemas de aprendizaje que presentan los alumnos. En este sentido propone varias etapas: recogida de informaciones relativas a los progresos y dificultades de aprendizaje; interpretación de estos datos; diagnóstico de los factores que causan las dificultades; y adaptación de las actividades educativas.

Desde otra perspectiva más práctica, diversas investigaciones se han enfocado en determinar, el nivel de desempeño docente vinculándolo directamente con el nivel de aprendizaje logrado en los 
alumnos. De acuerdo con León (2008), existe una relación positiva entre la competencia de los docentes y el aprendizaje del alumno. En este sentido, Glassick, Taylor y Maeroff (2003) consideraron que «no importa qué tan elocuente sea el desempeño del maestro, se requiere probar que ese desempeño ha tenido un impacto en el aprendizaje de los alumnos» (p. 55). Para probar que se han logrado esos aprendizajes, se debe hacer una evaluación del aprendizaje en los alumnos, en dos momentos, uno inicial, antes de la intervención del docente y otro al final para poder medir el valor agregado logrado en los alumnos como resultado de la actividad docente (Braun, 2005; Stewart, 2006). Sin embargo, esta forma de evaluación resulta muy controversial debido a que existen otros factores que determinan el nivel de competencia alcanzado por los alumnos, los cuales no pueden ser controlados por el docente o no están relacionados directamente a su intervención.

Ahora bien, para efectos de la medición del desempeño laboral, se deben considerar aspectos que influyen en el tipo de método, técnica e instrumento a emplear, como puede ser el tamaño y características de la población objetivo, los propósitos de la evaluación (medición de desempeño), presupuesto, recursos humanos o infraestructura. Tomando en cuenta que una evaluación a gran escala es estandarizada, lo que implica realizar un modelo complejo y cuidar la selección de variables. Regularmente, múltiples investigaciones optan por analizar la realidad separando las partes en componentes y establecen modelos desde perspectivas como la enseñanza eficaz (effective teaching) (Adegbile y Adeyemi, 2008; Good, Wiley y Florez, 2009) y parten de lo que sería un "buen docente" (Perrenoud, 1999; Danielson, 2013). Esto debido a que se facilita la conceptualización y operacionalización de las variables. En este sentido, Weaver (1948) considera que los problemas de simplicidad corresponden a situaciones que pueden ser descritas con pocas variables. Determina la importancia de las prácticas docentes para el aprendizaje del alumno, partiendo de que el fin último es que el alumno incremente sus conocimientos, habilidades y actitudes de acuerdo con los propósitos establecidos en el programa de trabajo.

Otros investigadores parten de que es posible comprender el proceso de enseñanza desde un modelo CIPP (Contexto-Insumo-Proceso-Producto). Se apoyan en el enfoque de sistemas (Garduño, 1999) y corrientes de calidad educativa (INEE, 2008 y 2005; Martínez y Coll, 2007). Dentro de un pensamiento sistémico y con un acercamiento lineal desde la fórmula «insumo-proceso-producto», la práctica docente entra en condición de insumo y proceso, el contexto se considera como un «escenario» y el aprendizaje como producto.

A decir de Garduño (1999), en la enseñanza-aprendizaje se puede hacer la distinción entre los insumos, procesos y productos. Por lo que, de cada uno, se pueden extraer sus atributos de manera absoluta, descriptiva o relacional-explicativa. La eficacia del proceso de enseñanza se puede expresar como un juicio de valor sobre un atributo o un conjunto de atributos. Este investigador identifica dos dimensiones de la evaluación: una absoluta-descriptiva y otra relacional-explicativa. En la primera, los juicios de valor se expresan sobre cualquiera de los componentes de los insumos, procesos, resultados o productos de manera aislada y en términos de propiedades. En la segunda se tiene como propósito determinar la relevancia, eficacia, efectividad, congruencia y eficiencia del docente.

Otra forma de aproximación, opuesta a la ciencia positivista con su paradigma de la simplicidad, linealidad y reducción, es la que establece que los sistemas y sus propiedades deben ser analizados en su conjunto, pero no por la suma de sus partes, sino a través de un todo integrado, en el que, dependiendo de su conformación final, se establece cómo se comportan las partes. Para ayudar a comprender cómo interactúan estos elementos, se entiende que el conjunto guarda estrechas relaciones entre sí y conforman un sistema más o menos estable y, normalmente, se enfoca en alcanzar algún tipo de objetivo (Bertalanffy, 1976). Por lo que no puede explicarse solo por el análisis de sus elementos constituyentes. Se entiende que es un sistema complejo y sus características solo pueden ser observadas desde la totalidad, por lo que sus propiedades no pueden reducirse a sus elementos. Un sistema complejo presenta una sensible dependencia de las condiciones iniciales. Los sistemas complejos tienen múltiples interacciones que determinan el producto final, se requiere contemplar, al mismo tiempo, diferentes representaciones del sistema.

En otro sentido, el ámbito educativo es un sistema abierto compuesto por subsistemas en los que interactúan diversos elementos, tanto internos como externos a la escuela, que están interactuando, determinando condiciones y circunstancias, que no son fijas, sino que varían en el tiempo, a las cuales debe responder el docente al realizar su labor. En este sentido, el enfoque multirreferencial ayuda a advertir sobre la complejidad de la práctica educativa docente para la evaluación y considera que se deben integrar diversos factores, hechos y situaciones (Ardoino, 2001). 


\section{Algunos referentes laborales}

En México, antes de la reforma educativa del año 2013, las relaciones laborales que se establecían entre los docentes y el Estado estaban reguladas por el Apartado B, del Artículo 123 de la Constitución Política de los Estados Unidos Mexicanos. Sin embargo, a partir de la implementación de la reforma educativa del año 2013, se creó un régimen de excepción laboral. Lo que implicó que, de acuerdo con las características laborales, se atiende su situación jurídica. Esto llevó a crear leyes exprofeso. Así, la Ley General del Servicio Profesional Docente (LGSPD) (DOF, 2013, 11 septiembre) estableció las condiciones para el ingreso, desempeño, reconocimiento y promoción docente.

De estos tipos de evaluaciones, el presente estudio, solo se enfoca en la evaluación de desempeño para la permanencia en el servicio para docentes de educación básica. La Ley del Servicio Profesional Docente (DOF, 2013, 11 septiembre) en su artículo 52 establece que las autoridades educativas y los organismos descentralizados deben evaluar el desempeño de los docentes en la educación que imparta el Estado. Para el caso de docentes de nuevo ingreso, el artículo 22 establece que, en una primera fase, el docente será contratado para un periodo de dos años que se podría considerar como de "prueba". En el caso de docentes de nuevo ingreso, al término del primer año escolar, presentan una autoevaluación diagnóstica y se les brindan los apoyos y programas pertinentes para fortalecer las capacidades, conocimientos y competencias requeridas. Al segundo año, presentan una evaluación de desempeño para la permanencia. En caso de que el personal no atienda a los apoyos y programas previstos; incumpla con la obligación de evaluación al término del periodo o se identifique su insuficiencia en el nivel de desempeño de la función docente, se da por terminado los efectos del nombramiento, sin responsabilidad para la autoridad educativa o para el organismo descentralizado.

Para los docentes que ya se encontraban en servicio al momento de aplicarse la reforma, deben presentar una evaluación de desempeño y en caso de resultar «No aptos» se les dará dos oportunidades más. El artículo 53 de la Ley General del Servicio Profesional Docente establece que en caso de que el personal no alcance un resultado suficiente en la tercera evaluación, se le cambiará de funciones. En ambos casos, se evaluará el desempeño del personal para determinar si en la práctica favorecen el aprendizaje de los alumnos y, en general, si cumple con las exigencias propias de la función.

De acuerdo con el documento Etapas, Aspectos, Método e Instrumentos (SEP, 2018, p. 12) para docentes del nivel de primaria, el docente debe presentarse a un proceso de evaluación que consiste en las siguientes etapas:

- Etapa 1. Informe de responsabilidades profesionales

- Etapa 2. Proyecto de enseñanza

- Etapa 3. Examen de conocimientos didácticos y curriculares.

En cuanto a los aspectos a evaluar, la evaluación del desempeño tiene como referente lo establecido en el documento Perfiles, Parámetros e Indicadores para docentes y técnicos docentes, aprobados por el Instituto Nacional para la Evaluación de la Educación (INEE), en este caso, para docentes del nivel de primaria. Dicho perfil está constituido por cinco dimensiones en las que se consideran los aspectos de la función docente establecidos en el artículo 14 de la LGSPD. 
Figura 1.

Evaluación del desempeño, docentes y técnicos docentes. Ciclo escolar 2018-2019. Perfil, parámetros e indicadores para docentes y técnicos docentes

\section{EDUCACIÓN PRIMARIA}

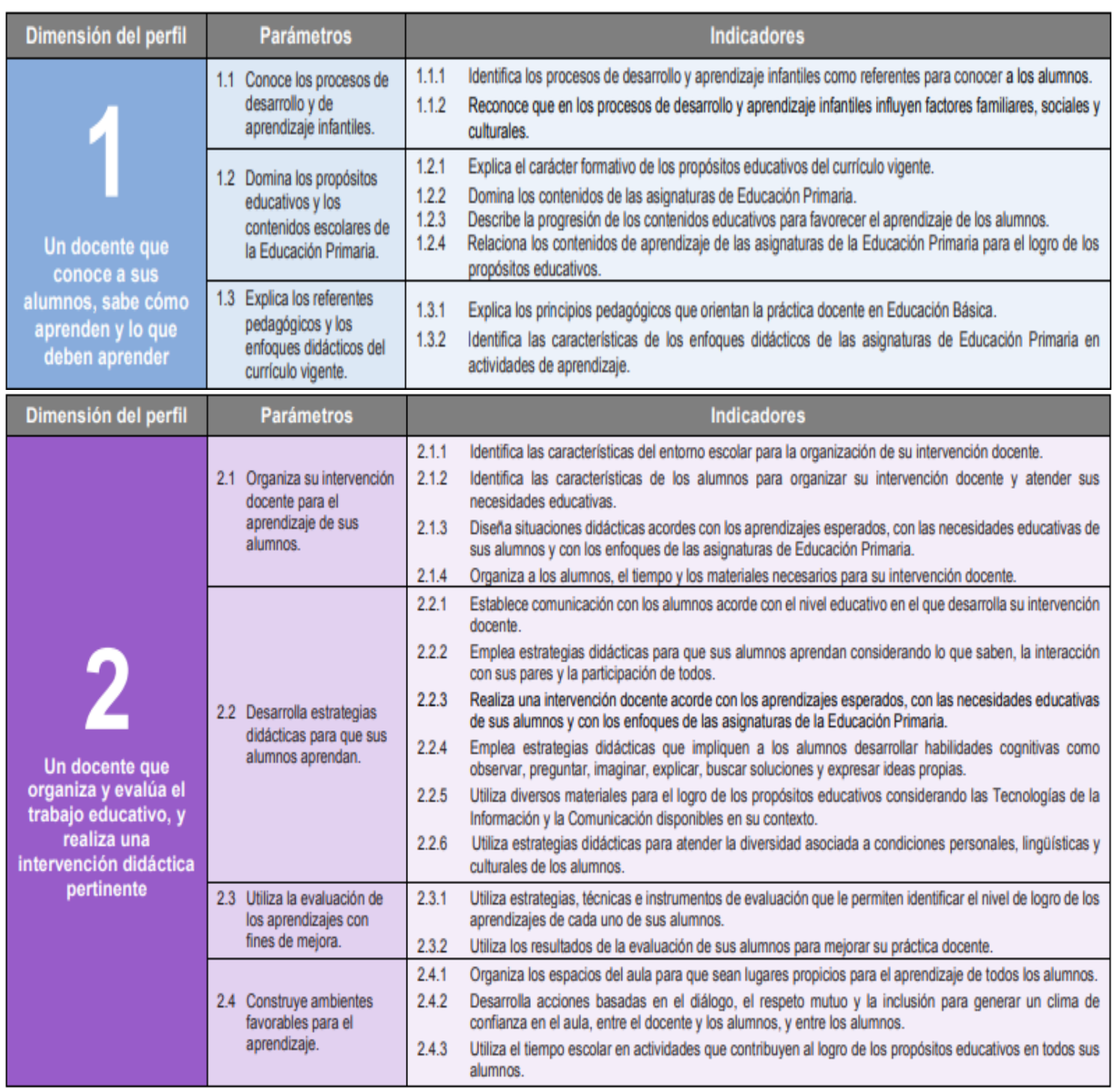




\begin{tabular}{|c|c|c|c|}
\hline Dimen & Parámetros & & Indicadores \\
\hline \multirow{3}{*}{$\begin{array}{l}\text { Un docente que se } \\
\text { reconoce como } \\
\text { profesional que } \\
\text { mejora continuamente } \\
\text { para apoyar a los } \\
\text { alumnos en su } \\
\text { aprendizaje }\end{array}$} & $\begin{array}{l}\text { 3.1 Reflexiona } \\
\text { sistemáticamente sobre } \\
\text { su práctica docente } \\
\text { como medio para } \\
\text { mejorarla. }\end{array}$ & \multicolumn{2}{|r|}{$\begin{array}{l}\text { 3.1.1 Identifica los aspectos a mejorar en su función docente como resultado del análisis de las evidencias de su } \\
\text { práctica. } \\
\text { 3.1.2 Utiliza referentes teóricos en el análisis de su práctica docente con el fin de mejorarala. } \\
\text { 3.1.3 Participa con sus pares en el análisis de su práctica docente con la finalidad de mejorarala. }\end{array}$} \\
\hline & $\begin{array}{l}3.2 \text { Emplea estrategias de } \\
\text { estudio y aprendizaje } \\
\text { para su desarrollo } \\
\text { profesional. }\end{array}$ & \multicolumn{2}{|r|}{$\begin{array}{l}\text { 3.2.1 Utiliza estrategias para la búsqueda, selección y uso de información proveniente de diferentes fuentes que } \\
\text { apoyen su desarrollo profesional. } \\
\text { 3.2.2 Utiliza la lectura de diferentes tipos de textos como una estrategia de estudio para fortalecer su desarrollo } \\
\text { profesional. } \\
\text { 3.2.3 Elabora textos orales y escritos con la finalidad de compartir experiencias y fortalecer su práctica } \\
\text { profesional. }\end{array}$} \\
\hline & $\begin{array}{l}3.3 \text { Utiliza diferentes medios } \\
\text { para enriquecer su } \\
\text { desarrollo profesional. }\end{array}$ & \multicolumn{2}{|r|}{$\begin{array}{l}\text { 3.3.1 Participa en redes de colaboración para fortalecer su desarrollo profesional. } \\
\text { 3.3.2 Utiliza el Consejo Técnico Escolar como un espacio para el aprendizaje y desarrollo profesional. } \\
\text { 3.3.3. Utiliza materiales impresos y las Tecnologlas de la Información y la Comunicación disponibles en su } \\
\text { contexto como medios para fortalecer su desarrollo profesional. } \\
\text { 3.3.4. Utiliza los espacios académicos como un medio para fortalecer su desarrollo profesional. }\end{array}$} \\
\hline Dime & \multicolumn{2}{|l|}{ Parámetros } & Indicadores \\
\hline \multirow{4}{*}{$\begin{array}{l}\text { Un docente que } \\
\text { asume las } \\
\text { responsabilidades } \\
\text { legales y éticas } \\
\text { inherentes a su } \\
\text { profesión para el } \\
\text { bienestar de los } \\
\text { alumnos }\end{array}$} & \multicolumn{2}{|c|}{$\begin{array}{l}4.1 \text { Considera los principios } \\
\text { filosóficos, los fundamentos } \\
\text { legales y las finalidades de la } \\
\text { educación pública mexicana } \\
\text { en el ejercicio de su función } \\
\text { docente. }\end{array}$} & $\begin{array}{l}\text { 4.1.1 Desarrolla su función docente con apego a los principios filosoficicos establecidos en el articulo tercero } \\
\text { constitucional. } \\
\text { 4.1.2 Aplica las disposiciones normativas vigentes que rigen su labor como docente de Educación Primara. } \\
\text { 4.1.3 Aplica la Normalidad Minima de Operación Escolar en su práctica docente cotidiana. }\end{array}$ \\
\hline & \multicolumn{2}{|l|}{$\begin{array}{l}\text { 4.2 Establece un ambiente } \\
\text { favorable para la sana } \\
\text { convivencia yla inclusión } \\
\text { educativa en su práctica } \\
\text { docente. }\end{array}$} & 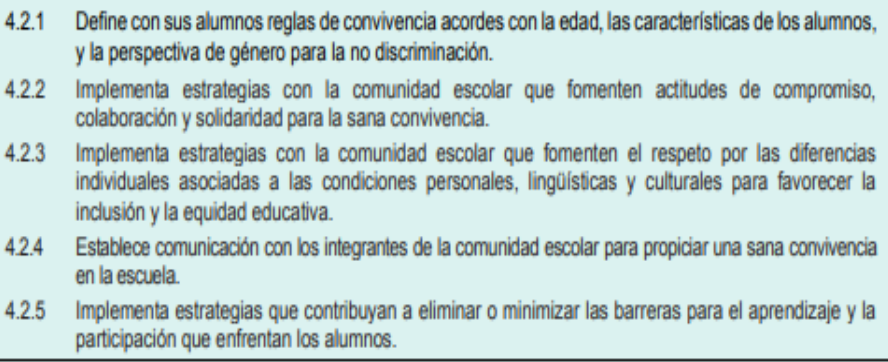 \\
\hline & \multicolumn{2}{|c|}{$\begin{array}{l}4.3 \text { Considera la integridad y } \\
\text { seguridad de los alumnos en } \\
\text { el aula y en la escuela. }\end{array}$} & $\begin{array}{l}\text { 4.3.1 Reconoce las implicaciones éticas y legales que tiene su comportamiento en relación con la integridad } \\
\text { y el sano desarrollo de los alumnos. } \\
\text { 4.3.2 Realiza acciones para la detección, canalización y seguimiento de casos de abuso o maltrato infantil } \\
\text { en el ámbito de su competencia. } \\
\text { 4.3.3 Aplica medidas preventivas para evitar enfermedades, accidentes y situaciones de resgo en el aula } \\
\text { yen la escuela en el ámbito de su competencia. } \\
\text { 4.3.4 ldentifica procedimientos para atender casos de emergencia que afectan la integridad y segunidad de } \\
\text { los alumnos como accidentes, lesiones, desastres naturales o violencia. }\end{array}$ \\
\hline & \multicolumn{2}{|c|}{$\begin{array}{l}4.4 \text { Demuestra altas expectativas } \\
\text { sobre el aprendizaje de todos } \\
\text { sus alumnos. }\end{array}$} & $\begin{array}{l}4.4 .1 \text { Reconoce la relación entre las expectativas que tiene sobre el aprendizaie de sus alumnos v sus } \\
\text { logros educativos. } \\
4.42 \text { Comunica a sus alumnos y a sus familias las altas expectativas que tiene acerca de sus aprendizajes, } \\
\text { considerando las capacidades que poseen. }\end{array}$ \\
\hline
\end{tabular}




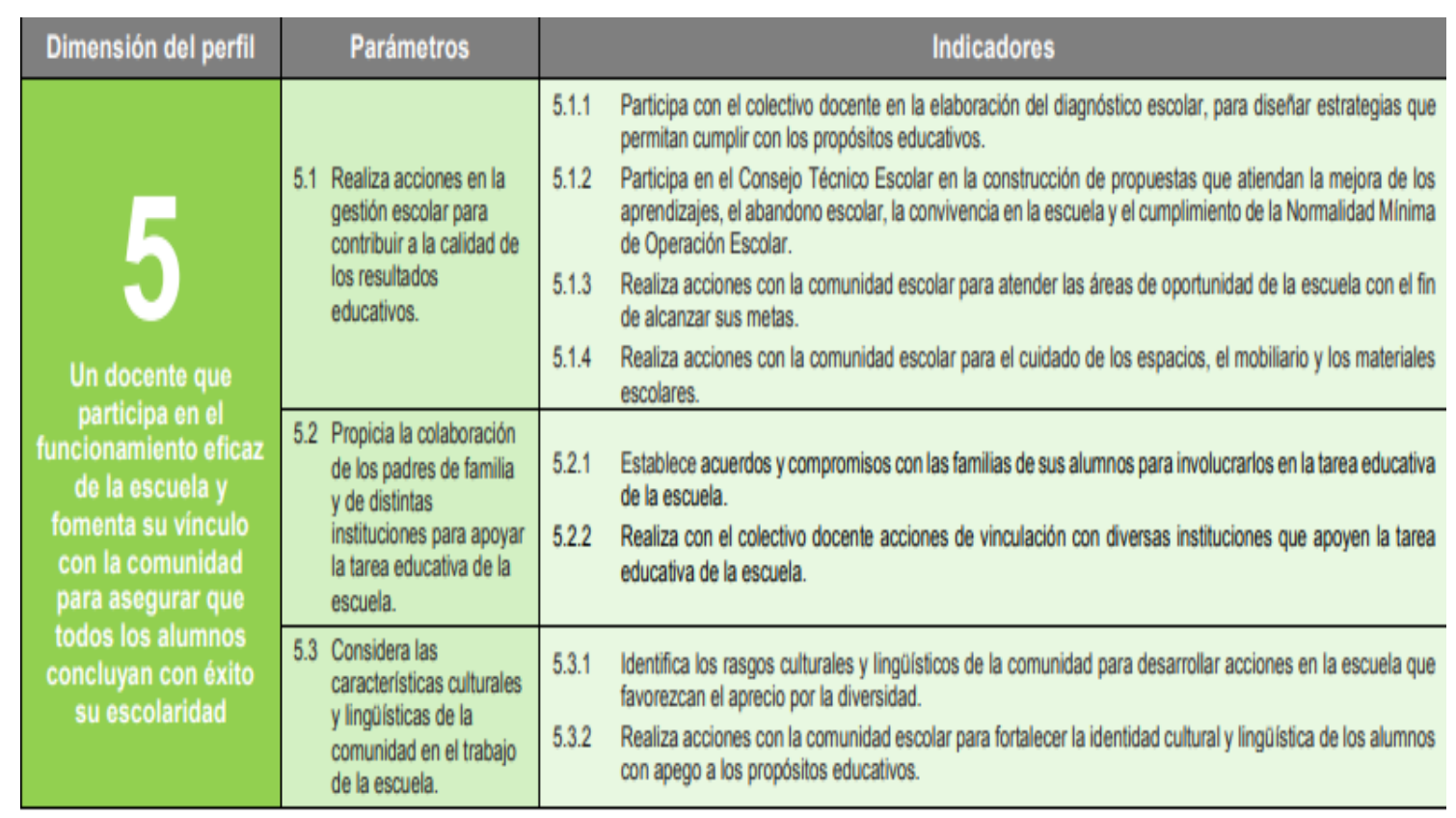

Fuente: SEP (2018, p. 32-36).

4. Tipos de instrumentos aplicados para la evaluación de desempeño para la permanencia

Como se indicó anteriormente, la evaluación de desempeño para la permanencia comprende los siguientes instrumentos: Informe de responsabilidades profesionales, Proyecto de Enseñanza y Examen de conocimientos didácticos y curriculares. Grosso modo, el primer instrumento se enfoca en determinar el grado de cumplimiento de las responsabilidades del docente en la escuela de acuerdo con la normatividad desde dos sentidos: desde su propia percepción y desde la de su inmediato superior; el segundo instrumento se enfoca en su capacidad para elaborar una planeación didáctica, su aplicación, evaluación y la reflexión sobre los resultados de la misma. Finalmente, el tercero, se aplica para determinar los conocimientos didácticos generales y específicos necesarios para enfrentar su práctica educativa cotidiana.

A continuación, se describirán las características de cada uno:

Informe de cumplimiento de responsabilidades profesionales. De acuerdo con el documento Etapas, aspectos, métodos e instrumentos (SEP, 2018, p. 7), se aplican dos cuestionarios de forma estandarizada, uno para el docente y otro para el superior inmediato del docente, son autoadministrables y se contestan en línea. Ambos cuestionarios tienen una estructura equivalente y abordan los mismos aspectos. Tienen el propósito de identificar en qué grado el docente cumple con las exigencias propias de su función. En estos instrumentos se evalúan principalmente las dimensiones 3, 4 y 5 del perfil del docente.

Según la Guía Técnica de la Autoridad Inmediata Superiorpara la Elaboración del Informe de Responsabilidades Profesionales del Personal Docente y Técnico Docente estos instrumentos se responden al término de su segundo año (SEP, 2017, p.16). Los cuestionarios miden, mediante una escala de tipo Likert, los siguientes aspectos: prácticas de convivencia en la comunidad escolar; prácticas de inclusión en la comunidad escolar; cumplimiento de la normativa vigente; vinculación con las familias e instituciones; trabajo colaborativo para la mejora escolar y mejora de la práctica profesional docente.

De acuerdo con los Criterios técnicos y de procedimiento para el análisis de los instrumentos de evaluación (DOF, 2017, octubre 23) la calificación se da con base en el escalamiento de las puntuaciones que resulten para cada uno de ellos, se integrará la información y se definirán cuatro categorías. Cada una de éstas tendrá asociada una cantidad de puntos que solo se adicionará a la puntuación total que obtenga el docente considerando el siguiente orden: NI: 0 puntos NII: 1 punto NIII: 2 puntos NIV: 3 puntos. En ningún caso, la omisión de alguno de los dos cuestionarios es causal de un resultado insuficiente. 
Examen de conocimientos y competencias didácticas. De acuerdo con el documento Etapas, aspectos, métodos e instrumentos (SEP, 2018), el docente o técnico docente debe dar cuenta de los conocimientos que posee para enfrentar su práctica educativa cotidiana, para lo cual, se aplica un examen de conocimientos de tipo curricular, disciplinar y sobre el proceso de enseñanza, se trata de enfocar qué «deben saber hacer» los docentes. El examen es estandarizado y está constituido por reactivos de cinco tipos: opción múltiple, jerarquización, relación de columnas, complementación, agrupamiento y cuestionamiento directo. El número de reactivos es de entre 100 y 120. En estos instrumentos se evalúan principalmente las dimensiones 1,3 y 4 del perfil del docente. Los principales aspectos a evaluar son sus capacidades para conocer los procesos de desarrollo y de aprendizaje; el dominio de los propósitos educativos y los contenidos escolares, explicar los referentes pedagógicos y los enfoques didácticos del currículo vigente, reflexionar sobre su práctica docente para mejorarla; emplear estrategias de estudio y aprendizaje para su desarrollo profesional; utilizar diferentes medios para enriquecer su desarrollo profesional; considerar los principios filosóficos, los fundamentos legales y las finalidades de la educación pública en el ejercicio de su función docente; establecer un ambiente favorable para la sana convivencia y la inclusión educativa en su práctica docente; considerar la integridad y, seguridad de los alumnos en el aula y en la escuela, y demostrar altas expectativas sobre el aprendizaje de todos sus alumnos.

Proyecto de enseñanza. El Proyecto de enseñanza es un instrumento que tiene el propósito de evaluar el desempeño del docente o técnico docente a través de una muestra de su práctica. En este instrumento, se evalúan principalmente las dimensiones 2, 3 y 4 del perfil del docente. Es la parte más importante de la evaluación ya que se asume que, debe "reflejar" lo que pasa en el aula. Consiste en elaborar una planeación didáctica, su aplicación y la reflexión que el docente hace en torno a su práctica. Se construye en tres fases: Momento 1. Elaboración del diagnóstico y de la planeación didáctica; Momento 2. Intervención docente; Momento 3. Respuesta a tareas evaluativas diseñadas para llevar a cabo un proceso de reflexión, con el apoyo de preguntas de andamiaje. Como producto de su intervención, los docentes seleccionarán tres evidencias considerando lo siguiente: que den cuenta de la organización, de la estrategia de evaluación y de la retroalimentación proporcionada a sus alumnos (SEP, 2018, p. 13). Es la etapa de evaluación que tiene mayor peso en la calificación final. Su evaluación mide la presencia de elementos que comprenden una planeación didáctica y sus cualidades.

5. Análisis de algunos aspectos normativos y técnicos de la evaluación de desempeño para la permanencia

Determinar la capacidad que tienen los instrumentos per ce para evaluar el desempeño de un docente es un tema polémico, sobre todo si se considera que la evaluación es de alto impacto (despido), ya que la práctica docente está afectada por múltiples factores, algunos de los cuales no son controlados por el docente. Por lo que resulta interesante, describir y analizar la experiencia de su implementación en México, algunas de sus limitaciones e inconsistencias, para finalmente realizar, de forma sucinta, algunas observaciones al respecto.

El trabajo se enfocó en los docentes de nuevo ingreso de educación básica, que presentaron su evaluación «Al término de su segundo año» y docentes que ya se encontraban en servicio al momento de la aplicación de la reforma, considerándose al grupo que presentaría su evaluación de «Tercera oportunidad». En primer lugar, se realizó un análisis textual con el propósito de determinar la consistencia entre la normatividad, leyes, lineamientos y documentos técnicos. En segundo lugar, se consultó el documento «Criterios técnicos para el desarrollo y uso de instrumentos de evaluación educativa, 2014-2015» (INEE, 2014, pp. 9-11) para el análisis técnico más específico. De éste, se tomó únicamente el siguiente criterio: a) definición del propósito y usos del instrumento de evaluación. El cual se refiere a los aspectos que se desean medir con el instrumento y la forma como serán utilizados los resultados de la evaluación. Esto implica determinar el propósito y la población objetivo; uso y alcance de sus resultados; tipo de instrumento, impacto o consecuencias de sus resultados; así como la modalidad y condiciones de administración o aplicación.

De manera general se asume, dentro de los textos consultados, que el propósito de la evaluación de los docentes es medir la calidad de la función. Se infiere, al consultar documentos de la Secretaría de Educación Pública (SEP), que la calidad es entendida como la «práctica [que] favorece el aprendizaje de los alumnos y... cumple con las exigencias propias de la función docente» (SEP, 2018, p.5). Para tal efecto, la SEP diseñó un modelo basado en perfiles, parámetros e indicadores, lo cuales tienen 
el propósito de servir como referentes. Desde La Ley General del Servicio Profesional Docente, su artículo 4 fracción XVII, determina como Marco General de lo que se debe entender como una Educación de Calidad:

«Al conjunto de perfiles, parámetros e indicadores que se establecen a fin (sic) de servir como referentes para los concursos de oposición y la evaluación obligatoria para el Ingreso, la Promoción, el Reconocimiento y la Permanencia en el Servicio Profesional Docente, con pleno respeto a los derechos constitucionales de los trabajadores de la educación» (DOF, 2013, 11 de septiembre).

En concordancia, los lineamientos que expide el Instituto Nacional para la Evaluación de la Educación (INEE) para llevar a cabo la evaluación del desempeño del personal que presenta su segunda y tercera oportunidad, en el ciclo escolar 2018-2019, en su artículo 6, establece que:

«La evaluación del desempeño de Docentes y Técnicos Docentes en Educación Básica, tiene la finalidad medir la calidad y los resultados de la función de desempeño, así como asegurar que se cumple con el perfil y el compromiso profesional que requiere un sistema escolar, para garantizar el derecho a la educación de calidad». (DOF, 2018, 7 mayo)

Al realizar la concordancia del proceso con la normatividad, se observó que existe una inconsistencia en cuanto a los propósitos de la evaluación en los textos que guían al docente en la aplicación de la evaluación. De acuerdo con el documento Etapas, Aspectos, Métodos e Instrumentos (EAMI) indica:

«[Es] una evaluación de carácter formativo. La Evaluación del Desempeño debe identificar logros y áreas de oportunidad de los docentes y técnico docente en servicio, con la finalidad, por un lado, de revelar los aspectos clave que deben fortalecer en su labor de manera oportuna y, por otro lado, mejorar sus prácticas de enseñanza mediante acciones de formación continua, tutoría y asistencia técnica necesarias que les permitan ubicar sus avances a lo largo de su trayectoria profesional. [Es] una evaluación que permita identificar los conocimientos y habilidades de los docentes y técnicos docentes en servicio y la experiencia adquirida a lo largo de su trayectoria profesional, además de identificar los rasgos fundamentales, necesarios y suficientes, de una práctica de enseñanza que conduzca al aprendizaje de los alumnos de Educación Básica [y] una evaluación que recupere información sobre las condiciones del contexto en que laboran los docentes y técnicos docentes que considere las características del entorno social y cultural y las condiciones específicas de la escuela y el aula, en que el docente y técnico docente desarrollan su práctica profesional de manera cotidiana.» (SEP, 2018, p. 6)

Por su parte, en el mismo sentido, el documento referente a los Perfiles, parámetros e indicadores se establece que:

«La evaluación del desempeño de docentes y técnicos docentes tiene como principal propósito que ellos y el sistema educativo cuenten con referentes comunes para la reflexión y el diálogo sobre las prácticas docentes que permiten un desempeño eficaz para que los alumnos logren los propósitos de la Educación Básica. Asimismo, los perfiles, parámetros e indicadores de desempeño de docentes y técnicos docentes permitirán orientar las acciones de formación continua para fortalecer su desarrollo profesional». (SEP, 2018, p. 7)

$\mathrm{Al}$ parecer, mientras las leyes y lineamientos establecen qué es una evaluación de desempeño para la permanencia, de tipo sumativa y de alto impacto laboral, en estos últimos documentos se enfatiza su función formativa, refiriéndose solo a la evaluación de primer año para los de nuevo ingreso y, de primera y segunda oportunidad para los que se encontraban en servicio al momento de la aplicación de la reforma. Esta situación crea incertidumbre pues no queda claro, cuál será su situación laboral de los docentes que presentan tal evaluación.

Por otro lado, las Guías académicas se diseñaron de forma estandarizada, por lo que no brindan información, de acuerdo con la disciplina o función de cada docente o el tipo de servicio en el que se encuentra inscrito. Con la reciente reforma de 2016 y su aplicación en los Planes y programas de 2018, algunos tipos de servicio o modelos educativos presentan una falta de actualización en sus modelos; planes y programas como es el caso de Telesecundaria o escuelas multigrado. Esto significa, que el 
docente trabaja en el aula con un modelo con el cual no va a ser evaluado. En una situación similar, se encuentran los docentes que prestan sus servicios en escuelas multigrado, ya que los instrumentos y Guías académicas están diseñadas para escuelas regulares y de organización completa.

En cuanto a la Definición del propósito y usos del instrumento de evaluación se aprecia una inconsistencia en cuanto a que se utiliza -al parecer- la misma la evaluación de desempeño, pero con diferentes propósitos de acuerdo con las circunstancias del docente:

- Si el docente que se encontraban con plaza al momento de la aplicación de la reforma aprueba la evaluación de desempeño, en su primera oportunidad, con un nivel de «Suficiente», por el hecho de ser aprobado sería evaluado nuevamente a los cuatro años. En este caso, la evaluación actuó como sumativa. En el caso de lo que aprueban como «Destacado», se le habilita para recibir incentivos económicos y/o promoción por asignación de horas adicionales (dependiendo el caso y condiciones institucionales) y deberá ser evaluado nuevamente a los cuatro años, en este caso, la evaluación actuó también como sumativa.

- Si el docente tiene una plaza al momento de la aplicación de la reforma y no aprueba la evaluación de desempeño, en su primera y segunda oportunidad, recibe asesoría y acompañamiento. En este caso la evaluación actúa como formativa y sumativa.

- Si el docente se encontraba en servicio antes de la reforma y no aprueba la evaluación de desempeño en su tercera oportunidad, es cambiado de funciones. En este caso, la evaluación actuó como sumativa y de alto impacto.

- Si el docente de nuevo ingreso se presenta a su evaluación de segundo año y resulta apto para el servicio, volverá a presentar otra evaluación a los cuatro años. En caso de no ser apto para el servicio, se terminaría la relación laboral. En ambos casos actuó como sumativa, pero en el segundo, además de sumativa, fue de alto impacto.

Tomando como base lo anterior y considerando la forma en que actualmente se presenta el proceso de evaluación, se infiere que se usan los mismos criterios para el diseño de los instrumentos en los diferentes momentos indicados. De ser así, los instrumentos deberían ajustarse de acuerdo con las circunstancias por las que pasa cada docente, es decir uno es el caso para los docentes que pueden volver a presentar la evaluación aun cuando han reprobado y otra, es la situación de los docentes que se encuentran en su última oportunidad, ya que tiene consecuencias laborales y políticas. Esto de conformidad con los Criterios técnicos para el desarrollo, uso y mantenimiento de instrumentos de evaluación del Instituto Nacional para Evaluación de la Educación (INEE) que establece lo siguiente:

«Determinar el propósito de la evaluación. Es la característica primordial del proceso, responde al para qué se va a evaluar y es el fundamento que da dirección a las actividades que se desarrollarán para construir el instrumento» (INEE, 2017, p. 7).

Además, se establece que se debe delimitar la población objetivo en función del propósito. Por lo que, se deben indicar las características de las personas a quienes está dirigida la evaluación, para las que son válidas las inferencias que se van a realizar y en caso necesario, se requiere determinar las diferentes subpoblaciones a las que está dirigida la evaluación (INEE, 2017).

En el mismo sentido, la población y subpoblaciones objetivo no están adecuadamente identificadas y caracterizadas, ya que no se discrimina la condición laboral y características de su trabajo, para determinar el tipo de instrumentos que se deben aplicar. Por otro lado, un problema detectado, no menos importante, es que no todos los docentes están frente a grupo, como se asume a priori. Algunos de ellos están comisionados en el sindicato o en la Secretaría de Educación Pública y otros cubren asignaturas que no tienen relación con su perfil profesional, por lo que no pueden cumplir adecuadamente con las distintas etapas de la evaluación, consecuentemente, se fomenta la simulación.

«Yo no estaba frente a grupo, porque estaba como coordinadora. Me dijeron que hiciera caso omiso a la notificación y un día antes de la evaluación me dijeron que siempre sí. Pues tuve que conseguir libretas de alumnos de la escuela. En realidad, fue una cuestión creativa lo que hice; me puse a redactar, pero no era cierto. Pero estuvo bastante bien, porque salí bien...» (INEE, 2016, p. 33) 
En otro orden de ideas, en cuanto a la modalidad de aplicación de la evaluación, se aplica en línea y/o formato electrónico por el Centro Nacional de Evaluación para la Educación Superior (CENEVAL). Este tipo de modalidad requiere el uso de computadoras y conectividad, lo cual ha generado algunos problemas para docentes que no tienen los recursos o competencias digitales.

Finalmente, se ha reportado, por parte de los docentes, que las sesiones son muy largas (examen de conocimientos, exámenes complementarios o de dominio de lengua indígena), para lo cual, se encontró que se han estado realizado cambios por parte de la SEP e INEE en los procesos y formas de aplicar los instrumentos buscando hacer menos pesadas las sesiones de evaluación. Por ejemplo, en el 2016 se revisaron los procesos de aplicación y se integraron dos fases del proceso de planeación didáctica en una y la forma de aplicación del examen complementario referido a lenguas indígenas también fue ajustada, ya que se requieren varias horas para su aplicación.

\section{Algunas observaciones sobre evaluación de desempeño para la permanencia}

A continuación, se expondrán algunas consideraciones que se derivaron del análisis de los aspectos anteriores. Sería conveniente que, para efectos de evaluación de desempeño para la permanencia, los instrumentos tomaran en cuenta, tanto aspectos empíricos, como teóricos. En un acercamiento al objeto de forma teórica, se buscaría llegar a una verdad contrastada con las observaciones empíricas. La combinación de observaciones de objetos empíricos y teóricos pudiera ser que eleve la validez de instrumentos.

Tratar de establecer cómo realiza un docente su práctica, no solo implica medir conocimientos, habilidades y productos, también se puede consultar a actores sociales clave que pueden dar cuenta del desempeño del docente en el trabajo. Esto es, en el Informe de cumplimiento de responsabilidades profesionales se debería dar, al director, mayor credibilidad y atribuciones, que a la fecha no tiene, ya que el director no puede tomar decisiones de alto impacto, en cuanto a la situación laboral de los docentes. Este problema fue detectado por la Organización para la Cooperación y Desarrollo Económicos (OCDE), la cual ha sugerido dotar a los directores de mayor autonomía para la toma de decisiones (OCDE, 2008). En el estudio Teaching and Learning International Survey, (TALIS) encontró, para el caso de México, que un porcentaje alto de directores considera que no tiene suficiente autonomía en la escuela. Declararon que es prioritario, en su trabajo de gestión escolar, dar mayor seguimiento a procesos burocráticos y no tienen injerencia en la contratación de profesores o la fijación de sus salarios (OCDE, 2013).

Con todo, un director debería tener la capacidad legal para generar sus equipos de trabajo y tomar decisiones en cuanto a despedir a un docente que no está acorde con las responsabilidades del puesto, para efectos, a su vez, de que él rinda cuentas sobre su propio desempeño. Esto implica implementar los instrumentos, mecanismos e instancias legales para que un docente pueda defender su causa como, por ejemplo: un consejo en el que participen autoridades educativas, sindicato y Asociación de Padres de Familia que, con evidencias presentadas por director y docente, al final decidan la permanencia del docente en la función.

Por otro lado, diseñar un modelo de evaluación del desempeño para la permanencia implica determinar el rendimiento ideal y la forma que debe adoptar la práctica docente en el aula. Según Camero (2008) se requiere: definir la situación a evaluar; especificar los propósitos de la evaluación; establecer los referentes evaluativos, lo que implica establecer a priori, los estándares, indicadores y seleccionar los instrumentos de medición que sean más adecuados.

Si tomamos como base el marco elaborado por Danielson (2013), lo comparamos con el de la SEP (2018) y los tratamos de acoplar, se puede observar, de manera general, que existe una relativa correspondencia entre ambas propuestas, se enfocan en el docente como el centro de todo el fenómeno; se prioriza el dominio del contenido teórico y práctico, con base en conocimiento de la pedagogía y la didáctica general y específica. En ambos casos, se considera importante crear espacios adecuados para el aprendizaje, la interacción social y la reflexión. Además, es de resaltar, en cuanto a la gestión del aprendizaje, la importancia del trabajo escolar comunitario que destacan.

Sin embargo, en el caso mexicano se resalta la importancia de conocer la normatividad, propio de un modelo de gestión burocrática y, por la otra parte, la propuesta de Danielson resalta la importancia de la interacción entre docente y alumnos, y el compromiso que muestran los alumnos para aprender. Esta dimensión es determinante para establecer condiciones más cercanas a la práctica cotidiana de un docente. Su desdibujamiento y falta en los Perfiles, Parámetros e Indicadores de la SEP, revela 
que, lo que se mide actualmente, no se comprueba en la realidad. La evaluación in situ permitiría observar la interacción y capacidad que tiene el docente para la resolución de problemas didácticos en el aula y las adecuaciones sobre su planeación didáctica, es decir su capacidad de retroalimentar su propio sistema de enseñanza de acuerdo con las conductas de sus alumnos.

Ahora bien, con el ajuste al modelo de evaluación del año 2016, se trató de incorporar a la evaluación, en el Proyecto Escolar, la dimensión contextual, en el cual el docente desarrolla su actividad. Este aspecto se mide en cuanto a la capacidad que tienen los docentes para incorporar el contexto a su proceso didáctico. Sin embargo, se debe considerar que el contexto afecta el proceso de enseñanza, de tal forma que, puede llegar a ser un elemento disruptivo, más que solo un elemento a incorporar en la planeación didáctica. Por lo que, actúa de dos formas, por un lado, es un elemento que debe ser incorporado dentro del proceso de enseñanza para facilitar el aprendizaje y, por otro, es un elemento externo que determina las condiciones en que un docente puede realizar su trabajo.

Asumiendo que, con la evaluación para el ingreso al Servicio Profesional Docente, se garantiza que los docentes tengan los conocimientos disciplinares y didácticos de forma mínima necesaria para llevar a cabo la función docente, una evaluación de desempeño, debería tener en cuenta otros aspectos. Por ejemplo, sería útil determinar en primer lugar, las condiciones en que desarrolla su trabajo. Ese contexto educativo presenta un tipo de ordenación del sistema que permite al docente desarrollar su labor en determinadas circunstancias. Por lo que, desde esta aproximación, se debe establecer una distancia con principios mecanicistas, reduccionistas y deterministas. Por lo que, el modelo insumoproceso-producto resulta poco útil.

Se entendería al sistema escolar como de tipo multifactorial, multicausal, dinámico, evolutivo, intra e interdependiente. Desde esta óptica se amplía el poder de abarcar las interacciones entre los diversos componentes de un sistema y de los sistemas entre sistemas y a varios niveles, solo para efectos de la inteligibilidad de las condiciones laborales.

En este sentido, es útil la teoría de sistemas para determinar un modelo más complejo de evaluación, como recomienda Ardoino (2001), lo que implica entender qué tipo de factores o variables interactúan a diferentes niveles y que inciden en el proceso de enseñanza, su importancia radica en que estos aspectos no pueden ser controlarlos por el docente, pero sí determinan el alcance de sus resultados. Se debe tomar en cuenta el identificar, medir y estimar el grado en que estos factores y variables influyen en el resultado del trabajo del profesor. De ahí la importancia de su medición para ponderarlos, al momento de evaluar al docente en su desempeño para efectos de permanencia.

Uno de los primeros problemas que se presentarían para resolver es la delimitación ontológica del contexto, puesto que, éste no es inherente al sistema, sino su determinación depende del investigador, en cuanto a cuáles son los más importantes. Considerando que el fenómeno se fundamenta en la interacción humana, se debe reconocer que todos los sistemas humanos son complejos y los hechos sociales se explican con otros hechos sociales. Desde una aproximación epistemológica, la evaluación de la práctica docente presenta una complejidad ontológica de estructura y proceso que tiene que ver con la variedad de formas posibles de ordenar los componentes y los posibles modos de interrelación.

Un sistema complejo presenta una sensible dependencia de las condiciones iniciales. Los sistemas complejos tienen múltiples interacciones que determinan el producto final, se requiere contemplar al mismo tiempo diferentes representaciones del sistema. Desde esta óptica se amplía el poder de abarcar las interacciones entre los diversos componentes y entre éstos, y el entorno a varios niveles, tratando de establecer los límites y circunstancias en cada fase, solo para efectos de la inteligibilidad de las condiciones laborales.

La aplicación de un modelo de evaluación compleja, implica entender qué tipo de factores o variables interactúan a diferentes niveles y que inciden en el proceso de enseñanza y se utilicen para ponderar al momento de evaluar el desempeño del docente para efectos de la permanencia. En este sentido, destacan las investigaciones de Mattews (2013) y otros investigadores (Rockwell, 2013; Jones, et al., 1987), que consideran determinante el trabajo de identificar, medir y estimar el grado en que estos factores y variables influyen en el resultado del trabajo del profesor, ya que sirven para ponderar su esfuerzo, conocimientos y experiencia aplicada.

Finalmente, desde esta perspectiva se entendería al proceso de enseñanza-aprendizaje como un subsistema dentro de un sistema, en el que se requiere identificar los diferentes subsistemas que se encuentran en interacción, las variables y el tipo de relaciones que se establecen entre ellas. En esta lógica, se entiende que el nivel de logro alcanzado por los alumnos dependerá de diversos aspectos. 
Consecuentemente, en el proceso, el docente sería desenfocado como responsable único de los resultados del sistema educativo. Solo se entendería como otra variable que interactúa con otras, a distintos niveles y con diferentes coeficientes de correlación. Así, se buscaría ponderar su actuación: en un primer subsistema estaría acotado a la triada: docente-alumno-contenido. En el que está en juego las características profesionales del docente (responsabilidad del docente) y las características de los alumnos (responsabilidad del alumno) y resultados (responsabilidad de varios actores sociales). Los siguientes niveles estarían conformados por las condiciones escolares y comunitarias (que incluye a la familia). Las variables o indicadores en estos sistemas tendrían un determinado peso dependiendo del aporte o afectación que hacen a la labor docente.

\section{Conclusiones}

$\mathrm{Al}$ analizar la consistencia que existe entre la normatividad para efectos de la evaluación de desempeño para la permanencia, se observó que existe concordancia entre leyes generales, tanto del INEE, como de la SEP. Sin embargo, cuando se comparó con los documentos técnicos y normativos que son de consulta para los docentes, existe una falta de claridad de las características de la evaluación de desempeño en las diferentes aplicaciones, ya que se está tratando de la misma forma, a todas la evaluaciones de desempeño que con motivo de segundo año para los de nuevo ingreso o para primera, segunda y tercera oportunidad para quien ya se encontraba en servicio al aplicarse la reforma, lo cual es un error técnico ya que sus propósitos, población y usos es diferente. Lo que confunde al maestro, quien no entiende las implicaciones de las distintas fases. Quizá esta situación se debe a que la evaluación de desempeño y sobre todo en lo referente a su aplicación para la permanencia es el tema más sensible de la puesta en marcha de la reforma educativa y lo que más inconformidades ha generado en los distintos estados de la república.

Esta situación ha llevado a distintos actores educativos y al mismo INEE a cuestionar el cumplimiento de los criterios técnicos en cuanto a la definición del propósito y usos del instrumento, entre otros aspectos. Al analizar los instrumentos únicamente en su fase de planeación, se encontró que presentan limitaciones en cuanto a su alcance, lo que representa un problema técnico cuando se habla de evaluación de alto impacto. Maestros plantearon que no miden el desempeño en el aula, sino solo se infiere el desempeño a partir de un informe de responsabilidades, un examen de conocimientos y en un proyecto de enseñanza. Estos instrumentos presentan fortalezas, pero también limitaciones que podrían afectar la validez para medir el desempeño para efectos de permanencia.

El resultado del instrumento Informe de cumplimiento de responsabilidades profesionales, no afecta la calificación, lo que le resta importancia al instrumento, solo podría otorgar al docente algunos puntos extra. Por tal situación, el docente tenderá a autocalificarse alto y en el caso del director, al no verse afectado o beneficiado de alguna forma, no tendrá inconveniente en llenarlo según sus intereses personales y no basado en el mérito. En cuanto al Proyecto de enseñanza, se asume que existe un alejamiento entre lo que sucede en el aula y lo que el docente presenta, ya que está basado en la coherencia de los elementos didácticos, evidencias y resultados que describe el docente y lo que finalmente reflexiona sobre su actividad profesional. En el proceso de revisión se busca identificar la presencia o no de elementos de forma coherente, lo que se evalúa con una rúbrica. El método y su instrumento presentan un límite ya que la coherencia entre lo planeado, la organiza el propio el docente y realiza una reflexión contra lo que resultó en su aplicación, restándole objetividad.

Finalmente, el Examen de conocimientos y competencias didácticas, si bien este tipo de instrumentos da cuenta de lo que el docente sabe y si sabe cómo podría aplicarse ese conocimiento, se aborda desde un conjunto de reactivos de opción múltiple, jerarquización, relación de columnas, complementación, agrupamiento y cuestionamiento directo, y es precisamente por el tipo de reactivos que resulta muy limitado inferir la capacidad y experiencia de una persona para resolver problemas de tipo pedagógico en el aula, ya que suelen tener problemas para discriminar y medir niveles cognitivos complejos, en suma son útiles para realizar una evaluación de ingreso, pero no una de desempeño.

En este caso, sería más conveniente aplicar los problemas de casos, reactivos que sí se incorporaron en un inicio de la reforma, pero que con las adecuaciones a la evaluación del año 2016 se excluyeron por las deficiencias en sus características técnicas. Esto dejó un vacío que requiere ser atendido para, de alguna forma, determinar el cómo se lleva a cabo la práctica docente en el aula. Por otro lado, debería marcar una mayor distancia con respecto a lo que se evaluó en el ingreso y la primera evalua- 
ción de desempeño, ya que una vez que un docente demostró que cuenta con la experiencia o conocimiento mínimo indispensable para ejercer la profesión, no se trataría de que explicara cómo se usa ese conocimiento, sino de tener una visión más amplia sobre lo que sucede en el aula y los resultados que alcanza.

Por otro lado, se debería indagar sobre aspectos contextuales para ampliar el nivel de validez de los instrumentos, al parecer se deja de lado, la observación empírica y condiciones en las que labora el docente. Se está considerando el contexto dentro del proyecto de enseñanza, lo cual solo indica que el docente, en su planeación didáctica, integra aspectos contextuales, sin embargo, para efectos de una evaluación de desempeño no se está considerando que las condiciones laborales, escolares y comunitarias afectan o ayudan en su labor, por lo que debería ser una puntuación externa que le dé una ponderación a lo obtenido por el profesor en los instrumentos.

Una evaluación in situ permite observar la interacción y capacidad que tiene el docente para la resolución de problemas didácticos en el aula y las adecuaciones sobre su planeación didáctica, es decir su capacidad de retroalimentar su propio sistema de enseñanza de acuerdo con las conductas de sus alumnos. De entre los cuales, algunos no tendrán las herramientas intelectuales mínimas o interés para que el docente puede desempeñarse adecuadamente.

La evaluación del desempeño del docente en el aula es una actividad compleja que requiere ser analizada desde diversos ángulos. Considerando que la evaluación de desempeño para efectos de permanencia laboral, implica decisiones de alto impacto para un docente, se requiere la combinación de métodos cuantitativos y cualitativos, así como de modelos teóricos y observaciones empíricas. Esto permitiría elevar de objetividad en el nivel medición y evaluación. Una clave para analizar esta situación puede ser el hecho de determinar, en primer lugar, qué es lo que es inherente al docente y, por otro, qué aspectos están determinados por otras circunstancias o factores externos. Por lo que, se debe partir de que el proceso de enseñanza es una variable que interactúa con otras y con factores, y que, pueden arrojar diferentes coeficientes de correlación.

Entender la dinámica interna, desde una visión de sistemas, permite aproximarnos a la medición del comportamiento docente en el aula, con base en la identificación, a distintos niveles, de los tipos e interacciones que se presentan. Permitiría un acercamiento al adecuado o inadecuado funcionamiento de alguna de sus partes, pero sin perder de vista que es parte de un todo. El abordaje sería como sistema y se observaría la variable «enseñanza» como un elemento más. De esta forma, el proceso de enseñanza, conjuntamente con otras variables arrojarían datos sobre cómo se presentan las interacciones y condiciones que están favoreciendo o no el proceso de enseñanza de forma aislada y en conjunto, lo que implica una aproximación desde una visión de una evaluación de desempeño para la permanencia como un sistema complejo.

\section{Referencias}

Adegbile, J. \& Adeyemi, B. (2008). Enhancing quality assurance through teacher's effectiveness. Educational Research and Review, 3(2), pp. 61-65.

Allal, L. (1980). Estrategias de evaluación formativa. Concepciones psicopedagógicas y modalidades de aplicación. Revista Infancia y Aprendizaje, 11, pp. 422. https://doi.org/10.1080/02103702.1980.10821803

Ardoino, J. (2001). La evaluación desgarrada: Entre un balance contable y el pleno ejercicio de una función crítica plural (multirreferencial). En M. Rueda, F. Díaz Barriga \& M. Díaz (Eds.), Evaluar para comprender y mejorar la docencia en la educación superior (pp. 19-35). México: UAM-UNAM-UABJO.

Bertalanffy, L. (1976). Teoría General de los Sistemas. México: FCE https://doi.org/10.1080/02103702.1980.10821803

Braun, H. (2005). Using Student Progress To Evaluate Teachers: A Primer on Value-Added Models. Princeton: Educational Testing Service.

Camero, M. (2008). La evaluación por competencias, mitos, peligros y desafíos. Educere: Revista Venezolana de Educación, (43), pp. 805-814. 
Coll, C. \& Solé, I. (2002). Enseñar y aprender en el contexto del aula. En C. Coll, A. Marchesi, \& J.Palacios (Comps.) Desarrollo Psicológico y Educación 2. Psicología de la Educación Escolar (pp. 357-386). Madrid: Alianza Editorial.

Danielson, Ch. (2013). The framework. Estados Unidos: The Danielson Group. Tomado de http://danielsongroup.org/framework/

DOF (2013, 11 septiembre). DECRETO por el que se expide la Ley General del Servicio Profesional Docente. Tomado de http:/ /www.dof.gob.mx/nota_detalle.php?codigo $=5313843 \&$ fecha $=11 / 09 / 2013$

DOF (2017, febrero 20). Lineamientos para la selección, capacitación y certificación de evaluadores del desempeño en Educación Básica y Media Superior para el ciclo escolar 2017-2018. LINEE-03-2017. Tomado de http:/ /www.dof.gob.mx/nota_detalle.php?codigo $=5472422 \&$ fecha $=20 / 02 / 2017$

DOF (2017, 23 octubre). Criterios técnicos y de procedimiento para el análisis de los instrumentos de evaluación, el proceso de calificación y la emisión de resultados para llevar a cabo la evaluación del desempeño del personal docente y técnico docente en Educación Básica en el ciclo escolar 2017-2018. Tomado de http://www.inee.edu.mx/images/stories/2014/Normateca/CRIT_DESMP_DOC_EB.pdf

DOF (2018, 7 mayo). Lineamientos para llevar a cabo la evaluación del desempeño del cuarto grupo de docentes y técnicos docentes, así como del personal con funciones de Dirección y Supervisión, y del personal que presenta su segunda y tercera oportunidad en Educación Básica en el ciclo escolar 2018-2019. LINEE-07-2018.Tomado de http:/ /www.dof.gob.mx/nota_detalle.php?codigo $=5521741 \&$ fecha $=07 / 05 / 2018$

Garduño, L. (1999). Hacia un modelo de evaluación de la calidad de instituciones de educación superior. Revista Iberoamericana de Educación, 21. Tomado de http://www.rieoei.org/rie21a06.htm

Glassick, C.; Taylor, M., \& Maeroff, G. (2003). La valoración del trabajo académico. México: UAM/ANUIES.

Good, T. L., Wiley, C. R. H., \& Florez, I. R. (2009). Effective Teaching: An Emerging Synthesis. En L.J. Saha \& A.G. Dworkin (Eds), International Handbook of Research on Teachers and Teaching (pp. 803815). New York: Springer. https://doi.org/10.1007/978-0-387-73317-3_51

INEE (2008). Análisis multinivel de la calidad educativa en México ante los datos de PIS A 2006. México: INEE

INEE (2014). Criterios técnicos para el desarrollo y uso de instrumentos de evaluación educativa, 2014-2015. Tomado de http://www.inee.edu.mx/images/stories/2014/criterios/Criterios_t $\%$ C3\%A9cnicos.pdf

INEE (2018). Educación para la democracia y el desarrollo de México: INEE.

INEE (2016). Evaluación del desempeño desde la experiencia de los docentes. México: INEE

León, M. (2008). Calidad docente y rendimiento escolar en Chile. (Tesis doctoral). Pontificia Universidad de Chile, Santiago de Chile.

Matthews, P. (2013, diciembre). Mejores escuelas: Estrategias para la acción en México. Ponencia en Seminario Evaluación Formativa del Desempeño Docente. Ciudad de México.

OECD (2008). Improving School Leadership Volume 1: Policy and Practice. Paris: OECD Publishing. https://doi.org/10.1787/9789264167995-lt

OECD (2013). Teaching and Learning International Survey TALIS 2013 Conceptual Framework. Otawa: IEA 
Rockwell, E. (octubre, 2013). Buenos maestros: no es fácil distinguirlos en la observación de la práctica. Seminario Evaluación Formativa del Desempeño Docente. Seminario dirigido por el Instituto Nacional para la Evaluacion de la Educación. Ciudad de México.

SEP (2017). Guia Técnica de la Autoridad Inmediata Superior para la Elaboración del Informe de Responsabilidades Profesionales del Personal Docente y Técnico Docente al término de su segundo año y del Personal con Funciones de Dirección al término de su periodo de inducción 2018. México: SEP.

SEP (2018). Perfiles, parámetros e indicadores. Evaluación del Desempeño. Docentes y Técnicos Docentes. Ciclo Escolar 2018-2019. Tomado de http://www.servicioprofesionaldocente.sep.gob.mx/content/ba/docs/2016/permanencia/parametros_indicadores/PPI_DOC_TECNICO_DOCENTES_080118.pdf

SEP (2018). Guia para la elaboración del Informe de cumplimiento de responsabilidades profesionales. México: SEP Tomado de http://servicioprofesionaldocente.sep.gob.mx/content/ba/docs/2015/permanencia/guias_academicas/Guia_15_GUIA_PARA_LA_ELABORACION_ICRP.pdf

Stewart, E. (2006). V alue-Added Modeling: The Challenge of Measuring Educational Outcomes. Carnegie Corporation. New York.

Weaver, W. (1948). Science and complexity. American Scientist (36), pp. 536-544. 